\title{
Title: Predicting Recovery and Outcome After Pediatric Stroke: Results from the International Pediatric Stroke Study
}

Running Title: Outcome and Recovery After Pediatric Stroke

Ryan J. Felling ${ }^{1 *}$, Mubeen F. Rafay ${ }^{2 *}$, Timothy J. Bernard ${ }^{3}$, Jessica L. Carpenter ${ }^{4}$, Nomazulu Dlamini $^{5,11}$, Sahar M. A. Hassanein ${ }^{6}$, Lori C. Jordan ${ }^{7}$, , Michael J. Noetzel ${ }^{8}$, Michael J. Rivkin ${ }^{9}$, Kevin A. Shapiro ${ }^{10}$, Mahmoud Slim ${ }^{5,11}$, Gabrielle deVeber ${ }^{5,11}$ on behalf of the International Pediatric Stroke Study Group

${ }^{1}$ Department of Neurology, Johns Hopkins University School of Medicine, Baltimore, MD

${ }^{2}$ Department of Pediatrics and Child Health, University of Manitoba, Children's Hospital Research Institute of Manitoba, Winnipeg, Manitoba, Canada

${ }^{3}$ Department of Pediatrics, University of Colorado, Aurora, CO

${ }^{4}$ Departments of Pediatrics and Neurology George Washington University Children's National Medical Center, Washington, D.C.

${ }^{5}$ Division of Neurology, Hospital for Sick Children, Toronto, Ontario, Canada

${ }^{6}$ Department of Pediatrics, Faculty of Medicine, Ain Shams University, Egypt

${ }^{7}$ Department of Pediatrics, Division of Pediatric Neurology, Vanderbilt University Medical Center, Nashville, TN

${ }^{8}$ Departments of Neurology and Pediatrics, Washington University School of Medicine, St. Louis, MO

${ }^{9}$ Departments of Neurology, Radiology, and Psychiatry, Boston Children's Hospital and Harvard Medical School, Boston, MA

${ }^{10}$ Department of Neurology, University of California San Francisco, San Francisco, CA

${ }^{11}$ Child Health Evaluative Sciences Program, Hospital for Sick Children, Toronto, Ontario Canada

\section{Correspondence:}

Ryan J. Felling, MD, PhD

200 N. Wolfe Street, Suite 2158

Baltimore, MD 21287

Phone: 1-410-955-4259

Fax: 1-410-614-2297

Email: rfelling@jhmi.edu

Title - 109 characters

Running Title -43 characters

Abstract - 249 words

Introduction -495 words

Discussion - 1495 words

Body of Manuscript - 4585 words

5 figures (no color), 3 tables

*These authors contributed equally to this manuscript. 


\begin{abstract}
Objective

To characterize predictors of recovery and outcome following pediatric arterial ischemic stroke, hypothesizing that age influences recovery after stroke.
\end{abstract}

\title{
Methods
}

We studied children enrolled in the International Pediatric Stroke Study between January 1, 2003 and July 31, 2014 with two-year follow-up after arterial ischemic stroke. Outcomes were defined at discharge by clinician grading and at two years by Pediatric Stroke Outcome Measure (PSOM). Demographic, clinical, and radiologic outcome predictors were examined. We defined changes in outcome from discharge to two years as recovery (improved outcome), emerging deficit (worse outcome), or no change.

\section{Results}

Our population consisted of 587 patients, including 174 with neonatal stroke and 413 with childhood stroke, with recurrent stroke in $5.8 \%$ of childhood patients. Moderate to severe neurological impairment was present in $9.4 \%$ of neonates vs $48.8 \%$ of children at discharge compared to $8.0 \%$ vs $24.7 \%$ after two years. Predictors of poor outcome included age between 28 days and one year (compared to neonates, OR 3.58, $\mathrm{p}<0.05$ ), underlying chronic disorder (OR $2.23, \mathrm{p}<0.05)$, and involvement of both small and large vascular territories (OR 2.84, $\mathrm{p}<0.05)$. Recovery patterns differed, with emerging deficits more common in children under one year of age $(\mathrm{p}<0.05)$.

\section{Interpretation}

Outcomes after pediatric stroke are generally favorable, but moderate to severe neurological impairments are still common. Age between 28 days and one year appears to be a particularly vulnerable period. Understanding the timing and predictors of recovery will allow us to better counsel families and target therapies to improve outcomes after pediatric stroke. 


\section{INTRODUCTION}

Pediatric arterial ischemic stroke (AIS) is an important cause of childhood neurologic disability. Although pediatric AIS is rare compared to adult stroke (incidence of 25 to 40 per $100,000^{1,2}$ live births and 1.2 to 8 per $100,000^{3-6}$ in older children), the consequences of pediatric stroke impact many more decades of life and impart substantial burdens on these children and their families. Management of AIS in children is largely extrapolated from the experience of adult stroke. This is problematic because the causes of AIS in children are significantly different from the typical cardiovascular risk factors in adults, ${ }^{7}$ and the immature brain provides a very different biological milieu than does the mature brain in its plasticity and recovery.

In prior studies, $76 \%$ of children with AIS leave the hospital with neurologic deficits. ${ }^{8}$ Neonates on the other hand frequently show little or no deficits at the time of stroke. ${ }^{9}$ Neurologic impairment persists in 30-50\% of children, at times even into adulthood. ${ }^{10-14}$ Sequelae include hemiparesis, speech and language deficits, cognitive impairment, behavioral problems, and epilepsy. These consequences frequently only become evident over time, as the child proceeds through advancing developmental stages. Early predictors of outcome would be useful for clinicians who are counseling parents and caregivers regarding recovery expectations after an acute stroke, yet despite tremendous advances in our capacity for neuroimaging and physiological monitoring, knowledge of outcome predictors remains limited. ${ }^{11,14-17}$

Predicting outcome after pediatric stroke relies not only on understanding how a particular stroke affects the structure and function of the brain, but also on how the brain recovers after stroke. A child's brain is a dynamic organ, still changing and developing well into adolescence. Many cite this plasticity as a basis for the expectation that outcomes will be better in younger patients, a theory based on Margaret Kennard's early observations. ${ }^{18,19}$ Advantageous plasticity following injury must compete with the impact of injury on normal development of neural networks, and the mantra of "younger brain, better recovery" may not be true. ${ }^{20-22}$ There is substantial clinical and preclinical data in adult stroke suggesting that a finite window exists for long-term recovery. ${ }^{23,24}$ While this may be longer in children, the extent of that window across childhood remains undefined.

Pediatric stroke has recently garnered more attention leading to the establishment of centers dedicated to improving the treatment of these patients. ${ }^{25}$ Defining predictors of outcome and recovery after stroke in children is important to appropriately select patients for therapies targeting neurological recovery. We used the International Pediatric Stroke Study (IPSS) to evaluate changes in neurological outcome between discharge and two years after acute ischemic stroke in a large cohort of children. We sought to define clinical and radiological predictors of outcome. We hypothesize that age influences stroke outcome based on the long held, but as yet unsubstantiated belief that younger brains recover better after injury. We studied recovery, defined as change in neurological status between discharge and two years to begin to define expectations for recovery after pediatric stroke.

\section{METHODS}

\section{The International Pediatric Stroke Study}


The IPSS is a prospective registry of children with AIS and cerebral sinovenous thrombosis (CSVT), with 87 enrolling centers in 24 different countries. Of these, 58 centers reported outcomes. Children are identified and consented at the sites by individual investigators, and clinical data are extracted from the medical record and entered into the database. The IPSS protocol is approved by the ethics committees of each enrolling IPSS site. The overall IPSS registry included 4,294 patients. We enrolled children from birth (>36 weeks gestation) to 18 years with a diagnosis of acute AIS made between January 1, 2003 and July 31, 2014 from IPSS centers reporting outcomes (Figure 1). Children with presumed perinatal stroke (i.e., chronic ischemic brain lesions that are identified on neuroimaging in childhood and presumed to have occurred in the perinatal period, but which were not identified acutely), concomitant CSVT, or non-ischemic stroke diagnoses were excluded. We also excluded children with death prior to hospital discharge. Symptomatic recurrent AIS is described if it occurred prior to follow-up. Children were included in the primary outcome analyses without regard to their recurrence status. Demographic data were collected including age, sex, center and country of enrollment. We categorized age as previously defined by the CDC National Center for Injury Prevention and $\mathrm{Control}^{26}$, with the modification that neonatal strokes (birth to 28 days of life) were considered as a separate age group. We collected clinical data into the IPSS registry from the acute stroke hospitalization health record including mode of presentation (abnormal level of consciousness, seizure (focal or generalized), headache, hemiparesis, visual field defect, speech abnormalities, ataxia, diplopia, vertigo, and papilledema) and risk factors for stroke including cardiac disease (congenital heart disease, prior surgery, acquired heart disease, isolated patent foramen ovale, other), arteriopathy (dissection, moyamoya, transient cerebral arteriopathy of childhood, postvaricella angiopathy, vasculitis, other), underlying chronic disorders (connective tissue disorders, iron deficiency anemia, sickle cell anemia, Downs syndrome, hematological malignancy, solid extracranial tumor, indwelling catheter, other), prothrombotic state (oral contraceptive use, Lasparaginase treatment, other), acute systemic illness (dehydration, sepsis, fever, acidosis, shock, anoxia/asphyxia, viral gastroenteritis, other), chronic head and neck conditions (aneurysm, arteriovenous malformation, migraine, MELAS, ventriculoperitoneal shunt, hemangioma/PHACES, other), acute head and neck conditions (sinusitis, otitis media, mastoiditis, meningitis, pharyngitis, head trauma, recent intracranial surgery, other). Radiological characteristics of the infarct were also recorded into the IPSS database including anterior or posterior circulation, single or multiple infarcts, small or large vessel distribution, and the presence of hemorrhagic conversion.

\section{Outcomes}

Outcomes were assessed at discharge and at two-year follow-up for all patients regardless of recurrence status during the follow up period. At discharge investigators entered a categorical deficit severity grade of normal, mild, moderate, or severe based on clinical examination findings. At two-year follow-up investigators entered the Pediatric Stroke Outcome Measure (PSOM), a validated standardized neurological examination encompassing five subscales: right sensorimotor, left sensorimotor, expressive language, comprehension, cognition/behavior. ${ }^{27}$ Each subscale is scored as follows: $0=$ normal, $0.5=$ minimal impairment with normal function, $1=$ mild impairment with slowed function, $2=$ severe impairment with at least one missing 
function (e.g. unable to pick up a coin with the fingers of one hand). The five subscales can be combined to yield a total PSOM severity classification system (PSOM-SCS) as follows: Normal $=0-0.5$ in all subdomains; Mild $=1$ in 1-2 subdomains and $<1$ in all remaining subdomains; Moderate $=1$ in $\geq 3$ subdomains or 2 in 1 subdomain and $<2$ in all remaining subdomains; Severe $=2$ in $\geq 2$ subdomains. ${ }^{28}$ These four categories parallel the clinician grading at discharge, enabling comparison of short and long-term outcomes. For logistic regression analysis the outcome was dichotomized into "good" (normal or mild) and "poor" (moderate or severe). "Recovery" was defined as any improvement in the categorical outcome between discharge and the two-year assessment. "Emerging deficit" was defined as worsening between these assessments.

\section{Statistical Analysis}

All statistical analyses were performed in Stata (Version 14.2, StataCorp, LLC, College Station, TX). Baseline characteristics were compared between the neonatal and childhood stroke groups using $X^{2}$ analysis. Nonparametric tests were used given the non-normal distribution of the data. We used the Wilcoxon-Mann-Whitney test to analyze differences in the outcome distributions between neonatal and childhood populations. The Wilcoxon signed rank test was used to analyze repeated outcome measures within each population. Predictors of dichotomous two year outcome were analyzed with logistic regression, with significance level $\alpha=0.05$. Individual predictors that met this threshold univariate analysis were included in the multivariable model. In the recovery analysis, the change in categorical outcome was calculated between discharge and two years after stroke. Differences in age groups were tested with Kruskal-Wallis equality of proportions rank test. We studied recurrence with time-to-event analysis with right censoring at the end of study follow-up (2 years post index stroke date). Hazard ratios (HR) and the corresponding 95\% CI for recurrent ischemic events were calculated using Cox proportional hazard models. Models were then adjusted by accounting for age and sex. Proportional hazards assumptions were assessed using the scaled Schoenfeld residuals.

\section{RESULTS}

\section{$\underline{\text { Sample Characteristics }}$}

Across the IPSS centers reporting outcomes, there were 3630 patients in the registry. Mortality by hospital discharge was reported in $1.5 \%$ of neonatal AIS patients and $3.3 \%$ of childhood AIS patients. After excluding death prior to discharge, AIS occurred in 2,578 patients of which 38 were excluded for concomitant CSVT. Two year outcomes were available for 587 AIS patients, representing our study population including 174 (31\%) neonatal AIS patients and 413 (69\%) childhood AIS patients (Figure 1). Table 1 provides the baseline characteristics of the study population. In the childhood-onset AIS group, the median age was 7.0 years (IQR 2.1 to 13.2 years). Each group had similar proportions with cardiac or acute systemic risk factors, while all other identified risk factors were more common in the childhood patients. Neonatal and childhood AIS presentation differed, with seizures being more common in the neonatal period (75\% vs 29\%, p<0.001) and hemiparesis being more common in older children (17\% vs 74\%, 
$\mathrm{p}<0.001)$. Other age-related differences included a predominance of left hemisphere infarcts in neonatal AIS ( $\mathrm{p}<0.001)$, and an increased frequency of posterior circulation involvement in childhood AIS ( $p<0.001)$. To assess the risk of bias in our study cohort compared to the larger cohort of IPSS patients without 2 year outcomes, we performed a number of $X^{2}$ analyses. Our study cohort was similar to the rest of the IPSS AIS registry in terms of sex $\left(X^{2}(1)=1.86\right.$, $p=0.172)$, age ( $t$-test, 0.273$)$, circulation involved $\left(X^{2}(2)=1.86, p=0.394\right)$, laterality $\left(X^{2}(2)=0.85\right.$, $\mathrm{p}=0.654)$, large or small vessel distribution $\left(\mathrm{X}^{2}(2)=5.30, \mathrm{p}=0.071\right)$, number of infarcts $\left(X^{2}(1)=0.028, p=0.867\right)$, or severity of neurological impairment at discharge $\left(X^{2}(3)=4.67\right.$, $\mathrm{p}=0.197)$. Risk factors did not significantly differ except that our sample had a higher percentage of patients with acute head and neck disorders $(20.3 \%$ vs $14.8 \%, \mathrm{p}=0.001)$.

\section{$\underline{\text { Summary of outcomes }}$}

Figure 2A shows the distribution of total PSOM scores in our neonatal and childhood stroke populations at two year follow up. In both age groups, the majority of scores were concentrated in the normal/mild range. Figure $2 \mathrm{~B}$ shows the range of categorized outcomes both at discharge and at two years, separated by age group. At the time of discharge, neonatal stroke outcomes were as follows: $67.8 \%$ normal, $22.8 \%$ mild, $8.1 \%$ moderate, $1.3 \%$ severe. Childhood stroke outcomes at discharge were as follows: $21.7 \%$ normal, $29.5 \%$ mild, $30.5 \%$ moderate, $18.3 \%$ severe. At two-year follow-up, neonatal stroke outcomes were as follows: $64.4 \%$ normal, $27.6 \%$ mild, $5.7 \%$ moderate, $2.3 \%$ severe. Childhood stroke outcomes at two-year follow up were as follows: $53.8 \%$ normal, $21.5 \%$ mild, $17.2 \%$ moderate, $7.5 \%$ severe. The distribution of outcomes was significantly different between children and neonates, both at discharge and at follow up with neonates having less severe outcomes at both timepoints $(\mathrm{p}<0.001)$. Figure $2 \mathrm{C}$ shows the two-year outcomes for three different domains of neurologic function measured by PSOM. This breakdown by domain was not available for the discharge assessment. In both age groups, sensorimotor problems were more prevalent than language or cognitive/behavioral problems, but differences between neonatal and childhood subgroups persisted ( $\mathrm{p}<0.05$ for motor and language, $\mathrm{p}<0.001$ for cognitive/behavioral).

We wanted to further understand the influence of age on outcome, and divided the childhood stroke patients into further age subgroups as defined previously by the CDC. ${ }^{25}$ Figure 3 illustrates the outcomes at two-year follow-up by age category at diagnosis. The total PSOM score was significantly different among age groups by Kruskal-Wallis equality-of-populations rank test, $X^{2}(5)=31.074, p<0.001$. Post hoc pairwise comparisons with Dunn-Bonferroni corrections indicated that the difference was significantly better in neonates compared to each of the three youngest childhood age groups (Figure 3A). Figure 3B indicates the SCS derived from the PSOM subdomain scores. Again there was a significant difference in two year outcomes among all age groups by Kruskal-Wallis, $X^{2}(5)=19.460, p=0.002$. Posthoc pairwise comparisons with Dunn-Bonferroni corrections indicated that outcomes were significantly better in the neonatal and 15-18 age groups compared to the young infant group, and between the neonatal and the 1-4 age group. Otherwise the age groups did not differ significantly from each other. These results indicate that a U-shaped curve exists in outcome at two years following stroke, with the worst outcomes in young infants having stroke beyond the neonatal period. Figures $2 \mathrm{C}$ and $3 \mathrm{C}$ illustrate severity scores of each subdomain of the PSOM by age group. In post hoc comparisons, sensorimotor outcomes differed between neonates and all childhood patients 
combined (Figure 2C), but when the childhood group was further divided the outcomes were not statistically different across the cohort (Figure 3C). In contrast, language and cognition did differ significantly across the cohort. When compared to the young infant group, post hoc comparisons showed better language outcomes in all other age groups except 1-4. The oldest children (10-14 and 15-18) also had significantly better outcomes than children aged 1-4. Cognitive outcomes were better in neonates compared to each childhood age group except for the oldest, and better in the 1-4 year, 10-14 year, and 15-18 year groups compared to the 5-9 year olds.

Our dataset represents a small fraction of the overall IPSS registry, and this raises the possibility of biased outcomes based on which patients return for follow-up. We performed sensitivity analyses using subsets of the IPSS registry to partially assess for this risk. Among 87 IPSS centers, 14 reported outcomes on at least $80 \%$ of their patients. These centers accounted for 407 AIS patients. When using our prespecified outcome time and restricting to these centers, we were able to account for $40 \%(n=161)$ of the patients. The outcomes were as follows: normal 99 (61\%); mild - 32 (20\%); moderate - 17 (11\%); severe - $13(8 \%)$. In addition we attempted to expand the inclusion even further by allowing a broader follow-up range of anything beyond one year after stroke, again restricting only to the high-reporting centers. This provided 255 patients $(63 \%)$. The outcomes for these patients were as follows: normal - $151(59 \%)$; mild - 51 (20\%); moderate $-36(14 \%)$; severe - $17(7 \%)$. Neither distribution was significantly different from our original dataset using all centers and restricting to the two year timepoint for perinatal and childhood strokes combined: normal - 310 (57\%); mild - 127 (24\%); moderate - 71 (13\%); severe $-32(6 \%)(p=0.420$ and $p=0.729$ by Wilcoxon rank sum test, respectively). To assess the influence of stroke recurrence on our overall outcomes we performed a sensitivity analysis comparing our entire cohort to the subset without any recurrent stroke prior to follow-up. Removing these patients did not change the neurologic outcomes or the associations identified in the prediction analysis.

\section{Predictors of outcome}

Table 3 summarizes the logistic regression analysis of predictors of outcome. In univariate analysis, any childhood age at stroke onset predicted worse outcome compared to neonatal age. In multivariable analysis, only age 1 month to 1 year at stroke onset remained predictive of worse outcome at two years following index stroke (OR 3.78, 95\% CI 1.16-12.3, p=0.028). Clinical presentation with hemiparesis or altered consciousness predicted poor outcome in univariate analyses but not in multivariable analysis. The only stroke risk factor that predicted poor outcome in multivariable analysis was the presence of an underlying chronic disorder (OR 2.15, 95\% CI 1.04-4.43, p=0.039). We also included radiological features of the stroke in our analysis. Univariate predictors of poor outcome included multiple infarcts and involvement of both small and large vessel territories. In multivariable analysis only the involvement of both large and small vessel territories was associated with poor outcome (aOR 2.89, 95\% CI 1.077.80, $\mathrm{p}=0.036)$.

\section{$\underline{\text { Recurrence }}$}


Recurrent AIS (symptomatic) did not occur prior to follow up in our neonatal AIS patients, but occurred in 34 childhood stroke patients $(5.8 \%)$, with a median interval of 33.5 days from incident stroke (IQR 10-171 days). Table 3 shows the Cox proportional HRs results for predictors of stroke recurrence. There were univariate associations with stroke recurrence for age, sex, vasculopathy, cardiac risk factors, underlying chronic disorders, and circulation involved. In multivariable models adjusted for age and sex, only vasculopathy was associated with stroke recurrence (HR 2.45, 95\% CI 1.16-5.43, $\mathrm{p}=0.019$ ).

\section{$\underline{\text { Recovery and emerging deficits }}$}

We wanted to analyze change in neurological status over the 2 year follow up period. This was limited by the fact that different outcome measures were available at discharge and follow up. At discharge a subjective assessment of the severity of neurological impairment was provided, while at follow-up the PSOM was reported, which we then classified into the SCS. In 20 patients both a subjective rating and a PSOM were reported at discharge, and in these patients we were able to directly compare the subjective discharge outcome with the SCS. In these 20 patients the outcome measures were matched in 6, and in the rest the subjective discharge outcome was rated as worse than the corresponding SCS (1 category worse in 12 patients and 2 categories worse in 2 patients). Therefore the subjective impression of outcome appeared to overestimate poor outcomes relative to the PSOM.

Figure 4 shows neurological change over time defined as recovery, no change, or emerging deficit. Overall 227 patients $(46 \%)$ demonstrated recovery over two years of follow up, while $189(39 \%)$ and $73(15 \%)$ demonstrated no change and emerging deficits, respectively. There was a significant difference among the age groups in terms of recovery status by Kruskal-Wallis equality-of-populations rank test, $X^{2}(5)=73.241, p=0.0001$. Posthoc pairwise comparisons with Dunn's test with Bonferroni corrections indicated significant differences between neonates or children 28 days to one year and each of the older age groups, with emerging deficits being more common in the younger groups. Neonates and children under one year were not significantly different from each other, nor were any of the older age groups significantly different from each other. Figure 5 illustrates the magnitude of change over the two-year follow up period and the relationship with initial status at discharge. When we excluded the 176 patients who were normal at discharge (and thus could not exhibit any recovery over time) and analyzed the remaining 313 patients, there was no significant association with age group and the demonstration of any degree of recovery over time; $X^{2}(5, N=313)=10.0, p=0.075$. We also analyzed the occurrence of full recovery to normal after two years. Of those categorized as mild at discharge $70 \%$ recovered fully to normal, compared to $47 \%$ of those categorized as moderate and only $12 \%$ of those categorized as severe. Similarly, there was no significant relationship between age group and full recovery for those patients with any neurological impairment at discharge, $\mathrm{X}^{2}(5, \mathrm{~N}=313)=8.91, \mathrm{p}=0.113$.

\section{DISCUSSION}

In this study two years after stroke we found normal outcome in the majority of patients and moderate to severe neurological impairment in $8.0 \%$ of neonatal AIS patients and $24.7 \%$ of childhood AIS patients. Recurrence prior to follow up occurred only in our childhood stroke 
patients and was associated with vasculopathy. This represents the largest study to date employing a standardized stroke-specific outcome measure in children. We illustrate a U-shaped relationship between age at stroke and outcome with young infants outside the neonatal period having the worst outcomes. Prior studies have included any neurological impairment as the outcome of interest, but this provides only a superficial understanding of the impact of stroke on children. By providing a range of severity based on impairment and function, our study better assesses the consequences of pediatric stroke. We also present data regarding recovery and emerging deficits. Understanding how children develop neurologically after stroke will be essential to finding more effective means of rehabilitation to improve outcomes.

Outcomes were favorable in most patients, with no abnormality or mild impairment with normal function in $92.0 \%$ of neonatal patients and $75.3 \%$ of childhood patients two years after stroke. The remaining $19.8 \%$ of our overall population had moderate to severe neurologic impairments, likely requiring substantial support for education and daily life activities. We used an updated definition of outcome severity in this study because prior studies have demonstrated that published definitions of PSOM summary scores (Table 1) overestimate poor outcomes by onethird. ${ }^{29}$ We wanted to emphasize the presence of disability, not just impairment, and to harmonize our scoring of pediatric stroke outcomes with adult outcomes where "unfavorable" is defined by a modified Rankin score (mRS) of 3-5, requiring an inability to independently carry out prior activities. Our results are concordant with some, but not all prior studies of outcome after pediatric stroke. Studies from Switzerland identified mRS scores $>2$ in 20-30\% after childhood stroke. ${ }^{13,14}$ A multicenter Canadian registry recently reported moderate-severe deficits in $32 \%$ of patients including both neonatal and childhood stroke based on clinician assessment. ${ }^{10}$ A single center study from London reported poor outcome (defined as deficits interfering with daily life) in $60 \%$ of their patients. ${ }^{30}$ Differences among studies could be due to outcome definition, variable follow up intervals, or inclusion criteria.

Our initial hypothesis that neonates would have better outcomes after stroke than older children was true both for overall outcomes and for different domains of neurologic function. Neonatal stroke patients often have normal neurological exams at presentation, but develop hemiparesis within the first year of life. Hence we believe the observation of better sensorimotor outcome in neonatal stroke patients as seen in Figure 2B is relatively robust. Conversely the better outcomes in speech, cognition, and behavior may represent an underestimate of deficits since these are more likely to emerge after two years of age. ${ }^{31,32}$ When examining the influence of age more specifically, we identified a U-shaped relationship between age and outcome as has been previously suggested. ${ }^{33,34}$ This trend was evident in all subdomains, although it was not significant for sensorimotor outcome. Even when considering other potential confounders early infancy (28 days to one year) predicted poorer outcome. We anticipated cardiac risk factors to play an important role in poor outcomes in this age group, but the present data did not support this. The presence of an underlying chronic disorder was predictive of poor outcome, possibly due to an abnormal neurologic baseline, but did not differ significantly among age groups. Early infancy is an important time of dynamic cerebral plasticity as neural circuits are established and remodeled, ${ }^{3532}$ and our data suggest that this may be a particularly vulnerable period. Based on clinical observations, two years is likely an insufficient duration to allow manifestation of cognitive and behavioral consequences in very young patients. Further understanding of agerelated outcomes for different domains of neurologic function will require longer term follow-up. 
We selected a two-year assessment interval after stroke with the assumption that we would have captured outcomes at a more stable timepoint after stroke when most recovery has occurred while minimizing the impact of emerging deficits during the highly active period of development from birth to age two years. This enabled us to eliminate variation in recovery due to variable time from stroke, but this also creates the possibility for selection bias as we only included a smaller subset of the overall IPSS AIS population who had two year outcomes available. In the IPSS standardized outcome data collection is variable depending on the feasibility at each site of re-assessing patients in clinic at serial intervals. However, our sample appears to be representative of the rest of the IPSS AIS population with respect to baseline patient characteristics based on our post hoc analysis. Given the consistency of our results with those previously reported for overall outcomes and this comparison, selection bias is unlikely.

Both preclinical data and observational studies in adults suggest a finite window for recovery after stroke, presumably due to a limited period of enhanced neuroplasticity. ${ }^{36-39}$ Many assume more potential for recovery in the child's brain, but we have little objective data to define such a window. One study suggested that the window for recovery in children is actually similar adults. ${ }^{40}$ Cooper et al. systematically examined motor recovery in neonates and children after AIS, finding that the frequency of sensorimotor impairments did not substantially change from 6 to 12 months after stroke, although their scores improved modestly. ${ }^{41}$ This group also showed an early PSOM assessment was more predictive of one year motor and adaptive behavior scores than age or lesion size. ${ }^{42}$ In our study there were surprisingly no differences among age groups in terms of degree of recovery at our detection capacity. This may imply that recovery mechanisms are common across all ages, or that there are competing processes in the youngest brains encompassing plasticity in some aspects and vulnerability in others. Understanding what is occurring during this critical period to allow recovery will be an important goal of future translational research, particularly as new treatment strategies emerge. The advent of noninvasive stimulation-based rehabilitative techniques such as transcranial direct current stimulation and transcranial magnetic stimulation appear promising as therapeutic modalities. ${ }^{43-45}$ Pharmacologic approaches to promote recovery are increasingly used in both clinical and research arenas. ${ }^{46,47}$ These treatments offer the potential to modulate neuroplasticity and hopefully augment spontaneous recovery, particularly if targeted to an optimal window which remains to be defined.

The IPSS registry includes only rudimentary descriptions of neuroimaging findings. Advanced neuroimaging analysis, including detailed lesion location and volume, will likely yield more robust prediction and should be a focus of future research. ${ }^{16}$ Such studies will be facilitated by current efforts to harmonize pediatric stroke imaging protocols. ${ }^{48,49}$ Our primary analysis compared the same outcome measure between groups. The secondary analyses of recovery, however, utilized 2 different outcome measures. Our comparison of the SCS and the subjective assessment at discharge in a subset patients suggests that the subjective assessment routinely overestimated discharge severity relative to the PSOM. Thus our recovery analyses may overestimate recovery while underestimating emerging deficits. Additionally, patients may have fluctuated within a category without being captured by our definitions of "recovery" and "emerging deficits". While we emphasized functional outcomes in this study, more granular 
measures of impairment in future studies would be necessary to really understand the capacity for true biological repair.

The most significant limitation of this study is the lack of standardized follow up among IPSS centers, resulting in only $21 \%$ of the AIS patients at reporting centers having outcome data at our prespecified recovery time. Our results should be interpreted with caution as this may bias the outcome distributions, in particular with regard to the most mildly and most severely affected patients as they may either not need to follow up or for whom follow up would be a challenge, respectively. Our subanalyses suggest that our dataset is representative of the overall IPSS registry in terms of baseline characteristics, risk factors, and severity at discharge. Additionally after relaxing our outcome timing criterion and restricting to centers with good consistency of outcome reporting, we did not see any difference in the distribution of outcomes. While this is encouraging it still cannot discount the risk of bias.

In summary, our data show good neurologic function in most patients two years after stroke, with significant disability in an important minority. Age is related to outcome, but not in a simple linear fashion. There may be periods of vulnerability perhaps related to critical periods of development. Emerging deficits were most common under one year of age, but recovery was otherwise not significantly different among age groups. This offers an important opportunity to better understand how recovery after stroke interacts with developmental plasticity to develop targeted therapies to improve outcomes. Future structured observational studies with standardized serial assessments across long durations of followup using the PSOM or other validated and sensitive measures of outcome are needed in pediatric stroke, to better define the critical period of recovery.

\section{Acknowledgements}

We would like to thank the National Institutes of Neurological Disorders and Stroke (K08NS097704 awarded to RJF) and the Auxilium Foundation for their support of this research.

\section{Author Contributions}

RJF, MAR, and GdV contributed to the conception and design of the study. RJF, MAR, TJB, JLC, ND, SMH, LCJ, MS, MJN, MJR, KS, and GdV contributed to the acquisition and analysis of the data. RJF, MAR, and GdV contributed to drafting of the text and preparation of the figures.

\section{Potential Conflicts of Interest}

None of the authors have any real or apparent conflicts of interest. 


\section{REFERENCES}

1. Lynch JK, Nelson KB. Epidemiology of perinatal stroke. Curr Opin Pediatr. 2001 Dec;13(6):499-505.

2. Schulzke S, Weber P, Luetschg J, Fahnenstich H. Incidence and diagnosis of unilateral arterial cerebral infarction in newborn infants. J Perinat Med. 2005;33(2):170-5.

3. Agrawal N, Johnston SC, Wu YW, Sidney S, Fullerton HJ. Imaging data reveal a higher pediatric stroke incidence than prior US estimates. Stroke; a journal of cerebral circulation. 2009 Nov;40(11):3415-21.

4. Fullerton HJ, Wu YW, Zhao S, Johnston SC. Risk of stroke in children: ethnic and gender disparities. Neurology. 2003 Jul 22;61(2):189-94.

5. Giroud M, Lemesle M, Gouyon JB, Nivelon JL, Milan C, Dumas R. Cerebrovascular disease in children under 16 years of age in the city of Dijon, France: a study of incidence and clinical features from 1985 to 1993. J Clin Epidemiol. 1995 Nov;48(11):1343-8.

6. Kleindorfer D, Khoury J, Kissela B, et al. Temporal trends in the incidence and case fatality of stroke in children and adolescents. Journal of child neurology. 2006 May;21(5):415-8.

7. Felling RJ, Sun LR, Maxwell EC, Goldenberg N, Bernard T. Pediatric arterial ischemic stroke: Epidemiology, risk factors, and management. Blood Cells Mol Dis. 2017 Mar 07.

8. Goldenberg NA, Bernard TJ, Fullerton HJ, Gordon A, deVeber G, International Pediatric Stroke Study G. Antithrombotic treatments, outcomes, and prognostic factors in acute childhoodonset arterial ischaemic stroke: a multicentre, observational, cohort study. Lancet Neurol. 2009 Dec;8(12):1120-7.

9. Kirton A, Armstrong-Wells J, Chang T, et al. Symptomatic neonatal arterial ischemic stroke: the International Pediatric Stroke Study. Pediatrics. 2011 Dec;128(6):e1402-10.

10. deVeber GA, Kirton A, Booth FA, et al. Epidemiology and Outcomes of Arterial Ischemic Stroke in Children: The Canadian Pediatric Ischemic Stroke Registry. Pediatric neurology. 2017 Apr;69:58-70.

11. deVeber GA, MacGregor D, Curtis R, Mayank S. Neurologic outcome in survivors of childhood arterial ischemic stroke and sinovenous thrombosis. Journal of child neurology. 2000 May;15(5):316-24.

12. Elbers J, deVeber G, Pontigon AM, Moharir M. Long-term outcomes of pediatric ischemic stroke in adulthood. Journal of child neurology. 2014 Jun;29(6):782-8.

13. Goeggel Simonetti B, Cavelti A, Arnold M, et al. Long-term outcome after arterial ischemic stroke in children and young adults. Neurology. 2015 May 12;84(19):1941-7.

14. Steinlin M, Roellin K, Schroth G. Long-term follow-up after stroke in childhood. Eur J Pediatr. 2004 Apr;163(4-5):245-50.

15. Lee J, Croen LA, Lindan C, et al. Predictors of outcome in perinatal arterial stroke: a population-based study. Annals of neurology. 2005 Aug;58(2):303-8.

16. Domi T, Vossough A, Stence NV, et al. The Potential for Advanced Magnetic Resonance Neuroimaging Techniques in Pediatric Stroke Research. Pediatric neurology. 2017 Apr;69:2436.

17. Studer M, Boltshauser E, Capone Mori A, et al. Factors affecting cognitive outcome in early pediatric stroke. Neurology. 2014 Mar 4;82(9):784-92.

18. Kennard MA. Relation of Age to Motor Impairment in Man and in Subhuman Primates. Arch Neurol Psychiatry. 1940;44(2):377-97. 
19. Kennard MA. Age and other factors in motor recovery from precentral lesions in monkeys. Am J Physiol. 1936;115:138-46.

20. Max JE, Bruce M, Keatley E, Delis D. Pediatric stroke: plasticity, vulnerability, and age of lesion onset. J Neuropsychiatry Clin Neurosci. 2010 Winter;22(1):30-9.

21. Golomb MR, Lo W. Do children recover better from arterial ischemic stroke than young adults? Neurology. 2015 May 12;84(19):1916-7.

22. Chapman SB, Max JE, Gamino JF, McGlothlin JH, Cliff SN. Discourse plasticity in children after stroke: age at injury and lesion effects. Pediatric neurology. 2003 Jul;29(1):34-41.

23. Zeiler SR, Krakauer JW. The interaction between training and plasticity in the poststroke brain. Current opinion in neurology. 2013 Dec;26(6):609-16.

24. Murphy TH, Corbett D. Plasticity during stroke recovery: from synapse to behaviour. Nature reviews Neuroscience. 2009 Dec;10(12):861-72.

25. Bernard TJ, Rivkin MJ, Scholz K, et al. Emergence of the primary pediatric stroke center: impact of the thrombolysis in pediatric stroke trial. Stroke; a journal of cerebral circulation. 2014 Jul;45(7):2018-23.

26. National Center for Injury Prevention and Control CuW. 10 Leading Causes of Death by Age Group, United States. 2016; Available from:

https://www.cdc.gov/injury/wisqars/LeadingCauses.html.

27. Kitchen L, Westmacott R, Friefeld S, et al. The pediatric stroke outcome measure: a validation and reliability study. Stroke; a journal of cerebral circulation. 2012 Jun;43(6):1602-8.

28. Slim M, Dlamini N, Fox C, et al. Outcome Severity Assessment with the Pediatric Stroke Outcome Measure (PSOM): Validation of a Novel Classification System. 47th National Meeting of the Child Neurology Society; Chicago, Il: Ann Neurol; 2018. p. S406.

29. Bulder MM, Hellmann PM, van Nieuwenhuizen O, Kappelle LJ, Klijn CJ, Braun KP.

Measuring outcome after arterial ischemic stroke in childhood with two different instruments. Cerebrovasc Dis. 2011;32(5):463-70.

30. Ganesan V, Hogan A, Shack N, Gordon A, Isaacs E, Kirkham FJ. Outcome after ischaemic stroke in childhood. Developmental medicine and child neurology. 2000 Jul;42(7):455-61.

31. Westmacott R, Askalan R, MacGregor D, Anderson P, Deveber G. Cognitive outcome following unilateral arterial ischaemic stroke in childhood: effects of age at stroke and lesion location. Developmental medicine and child neurology. 2010 Apr;52(4):386-93.

32. Westmacott R, MacGregor D, Askalan R, deVeber G. Late emergence of cognitive deficits after unilateral neonatal stroke. Stroke; a journal of cerebral circulation. 2009 Jun;40(6):2012-9.

33. Goodman R, Yude C. IQ and its predictors in childhood hemiplegia. Developmental medicine and child neurology. 1996 Oct;38(10):881-90.

34. Allman C, Scott RB. Neuropsychological sequelae following pediatric stroke: a nonlinear model of age at lesion effects. Child neuropsychology : a journal on normal and abnormal development in childhood and adolescence. 2013;19(1):97-107.

35. Semple BD, Blomgren K, Gimlin K, Ferriero DM, Noble-Haeusslein LJ. Brain development in rodents and humans: Identifying benchmarks of maturation and vulnerability to injury across species. Progress in neurobiology. 2013 Jul-Aug; 106-107:1-16.

36. Cramer SC. Repairing the human brain after stroke: I. Mechanisms of spontaneous recovery. Annals of neurology. 2008 Mar;63(3):272-87. 
37. Jorgensen HS, Nakayama H, Raaschou HO, Vive-Larsen J, Stoier M, Olsen TS. Outcome and time course of recovery in stroke. Part II: Time course of recovery. The Copenhagen Stroke Study. Archives of physical medicine and rehabilitation. 1995 May;76(5):406-12.

38. Krakauer JW, Carmichael ST, Corbett D, Wittenberg GF. Getting neurorehabilitation right: what can be learned from animal models? Neurorehabilitation and neural repair. 2012 Oct;26(8):923-31.

39. Felling RJ, Song H. Epigenetic mechanisms of neuroplasticity and the implications for stroke recovery. Experimental neurology. 2015 Jun;268:37-45.

40. Kim CT, Han J, Kim H. Pediatric stroke recovery: a descriptive analysis. Archives of physical medicine and rehabilitation. 2009 Apr;90(4):657-62.

41. Cooper AN, Anderson V, Hearps S, et al. Trajectories of Motor Recovery in the First Year After Pediatric Arterial Ischemic Stroke. Pediatrics. 2017 Aug;140(2).

42. Cooper AN, Anderson V, Hearps S, et al. The Pediatric Stroke Outcome Measure: A predictor of outcome following arterial ischemic stroke. Neurology. 2018 Jan 30;90(5):e365-e72. 43. Kirton A, Andersen J, Herrero M, et al. Brain stimulation and constraint for perinatal stroke hemiparesis: The PLASTIC CHAMPS Trial. Neurology. 2016 May 3;86(18):1659-67. 44. Kirton A, Ciechanski P, Zewdie E, et al. Transcranial direct current stimulation for children with perinatal stroke and hemiparesis. Neurology. 2017 Jan 17;88(3):259-67.

45. Gillick BT, Kirton A, Carmel JB, Minhas P, Bikson M. Pediatric stroke and transcranial direct current stimulation: methods for rational individualized dose optimization. Frontiers in human neuroscience. 2014;8:739.

46. Benders MJ, van der Aa NE, Roks M, et al. Feasibility and safety of erythropoietin for neuroprotection after perinatal arterial ischemic stroke. The Journal of pediatrics. 2014 Mar;164(3):481-6 e1-2.

47. Chollet F, Tardy J, Albucher J-F, et al. Fluoxetine for motor recovery after acute ischaemic stroke (FLAME): a randomised placebo-controlled trial. The Lancet Neurology. 2011;10(2):123-30.

48. Lee S, Mirsky DM, Beslow LA, et al. Pathways for Neuroimaging of Neonatal Stroke. Pediatric neurology. 2017 Apr;69:37-48.

49. Mirsky DM, Beslow LA, Amlie-Lefond C, et al. Pathways for Neuroimaging of Childhood Stroke. Pediatric neurology. 2017 Apr;69:11-23. 


\section{FIGURE LEGENDS}

Figure 1. IPSS patient breakdown and inclusion criteria. Patients were excluded if they had venous thrombosis, presumed perinatal stroke (PPERI), or a stroke syndrome other than AIS. Among the AIS patients we included only patients who had a PSOM assessment at 2 years after stroke, indicated by the bold text in the flow chart. ${ }^{*}=$ Overall IPSS registry included 4,294 patients.

Figure 2. (A) Frequency distribution of total PSOM scores in this study population. Scores for childhood stroke patients are shown in black $(\mathrm{n}=413)$ and scores for neonatal stroke patients are overlayed in white $(\mathrm{n}=174)$. (B) Categorical outcomes at discharge (based on clinician grade) and 2 years (based on SCS). (C) Categorical outcomes at 2 years based on subdomain scores of the PSOM. ${ }^{*}=\mathrm{p}<0.05,{ }^{* *}=\mathrm{p}<0.005$ by Wilcoxon-Mann-Whitney.

Figure 3. Outcomes at two-year follow-up. (A) Total PSOM score by age group. Overall $\mathrm{p}<0.001$ by Kruskal Wallis test. Posthoc analyses were done with Dunn Bonferroni pairwise comparisons. (B) Categorization of the PSOM score subdomains (SCS). Overall there was a significant difference among age groups by Kruskal Wallis, $\mathrm{p}=0.002$. Posthoc analyses were done with Dunn Bonferroni pairwise comparisons. (C) Subdomain severity scores by age category (for each subdomain, the age groups and severities are presented as in panel B). There was an overall difference for both language and cognitive behavioral domains by Kruskal Wallis, $\mathrm{p}<0.01$. Posthoc Dunn Bonferroni pairwise comparisons: *, $\mathrm{p}<0.05 ;{ }^{* *}, \mathrm{p}<0.01,{ }^{* * *}, \mathrm{p}<0.001$

Figure 4. Recovery status by age group. Recovery indicates any improvement in categorical severity at two years compared to discharge. Emerging deficit indicates any worsening in categorical severity at two years compared to discharge. ${ }^{*}=\mathrm{p}<0.05$ by Kruskal Wallis posthoc pairwise comparisons.

Figure 5. Recovery and emergence of new deficits at 2 years after stroke. Each point represents an individual patient and are jittered around 1 unit intervals to allow better visualization of the discrete data points. The dotted reference lines indicate the status at discharge, and points above the reference line have shown recovery, while points below the reference line have shown emerging deficits. (A) Normal neurologic status at discharge. (B) Minimal neurological impairment at discharge. (C) Mild neurological impairment at discharge. (D) Moderate to severe neurological impairment at discharge. There were no significant differences among the different age groups in terms of the degree of recovery or emerging deficits. 